Case Report

\title{
Ominous Occurrence of Spinal Intradural Primary Malignant Peripheral Nerve Sheath Tumor Four Decades following Radiation Therapy for Testicular Seminoma
}

\author{
Osmond C. Wu, ${ }^{1}$ Berje H. Shammassian, ${ }^{1}$ Arunit J. S. Chugh ${ }^{(D)},{ }^{1}$ Aparna Harbhajanka, \\ and Manish K. Kasliwal ${ }^{1}$ \\ ${ }^{1}$ Department of Neurological Surgery, University Hospitals Cleveland Medical Center, Cleveland, OH, USA \\ ${ }^{2}$ Department of Pathology, University Hospitals Cleveland Medical Center, Cleveland, OH, USA \\ Correspondence should be addressed to Arunit J. S. Chugh; arunit.chugh@uhhospitals.org
}

Received 16 August 2019; Accepted 27 November 2019; Published 28 January 2020

Academic Editor: Dominic B. Fee

Copyright $(92020$ Osmond C. Wu et al. This is an open access article distributed under the Creative Commons Attribution License, which permits unrestricted use, distribution, and reproduction in any medium, provided the original work is properly cited.

Primary intradural malignant peripheral nerve sheath tumor (MPNST) is an extremely rare diagnosis and is associated with an extremely poor prognosis. A 77-year-old man diagnosed with an intradural MPNST, more than 40 years after radiation for a testicular seminoma, is reported. Intradural MPNSTs of the spine outside the setting of neurofibromatosis is extremely rare and can masquerade as common benign nerve sheath tumors, on imaging. An older age at presentation with short duration of symptoms and prior regional radiation treatment encompassing the spine in the treatment field regardless of remoteness should alert the oncologists and neurosurgeons to the possible existence of this rare and aggressive tumor, as the management, and overall prognosis of this tumor is distinctly different compared to the usual intradural spinal tumors.

\section{Introduction}

Malignant peripheral nerve sheath tumors (MPNSTs) are aggressive, locally invasive rare soft tissue sarcomas arising from peripheral nerves that originate from Schwann cells or pluripotent cells of neural crest origin [1,2]. MPNSTs represent $3-10 \%$ of all soft tissue sarcomas, with an overall incidence of $0.001 \%$ in the general population that peaks in the seventh decade [2-4]. In patients with neurofibromatosis type 1 (NF1), the annual incidence is 1.6 per 1000 with a lifetime risk of $8-13 \%$ [5]. Prior radiation exposure is another important risk factor, with a reported incidence of radiation induced MPNST ranging from $5.5-11 \%$ of patients [6]. MPNSTs are usually located in the extremities, trunk, and head, and neck. Spinal MPNSTs, however, are exceedingly rare. Table $1 \mathrm{com}-$ prises a list of the spinal MPNSTs in the literature. While there are a few instances of spinal and paraspinal MPNST following radiation for testicular seminomas, all the cases reported occurred within 7-10 years following radiation and were mainly extradural in location [7-10]. An intradural spinal MPNST with subsequent intracranial leptomeningeal metastasis diagnosed forty years after radiation is presented in this report with a pertinent review of the literature.

\section{Case Report}

2.1. History and Presentation. A 77-year-old man with past medical history significant for esophageal adenocarcinoma as well as testicular seminoma that was treated with conventional external bean radiation therapy (EBRT) almost 40 years back presented with three months of left hip and buttock pain in addition to left foot weakness. He had no history or clinical stigmata of neurofibromatosis.

A magnetic resonance imaging (MRI) of the lumbar spine with and without gadolinium demonstrated a homogenously enhancing, well demarcated intradural extramedullary neoplasm (Figure 1). An initial MRI of the cervical and thoracic spine with and without gadolinium was negative for additional lesions. While a diagnosis of benign nerve sheath tumor was suspected given typical radiological appearance, a metastatic lesion was also considered in the differential because of prior 


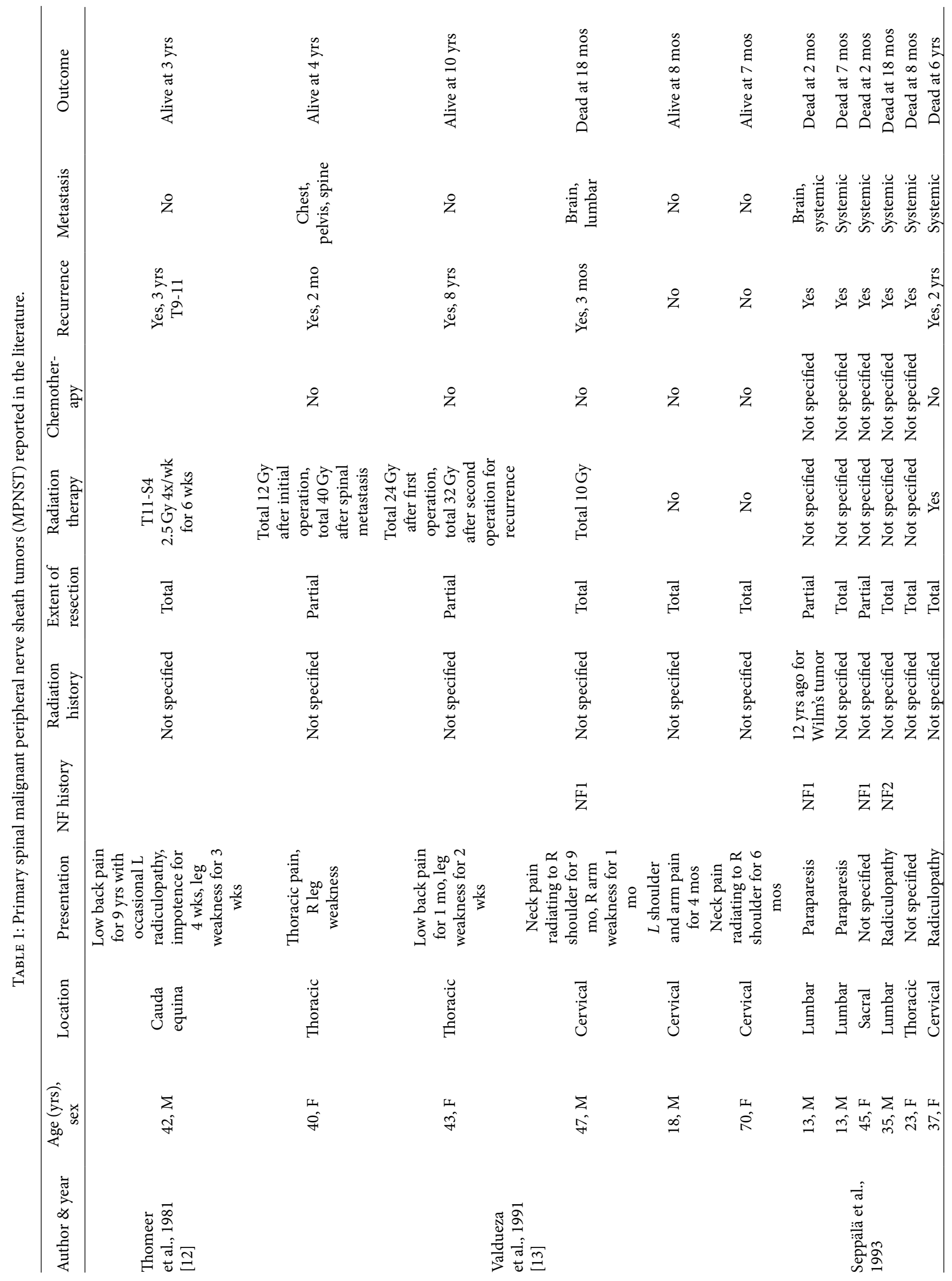




\begin{tabular}{|c|c|c|c|c|c|c|c|c|c|c|c|c|c|c|c|c|c|}
\hline 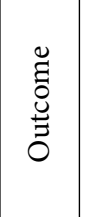 & 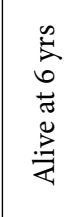 & 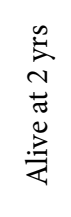 & 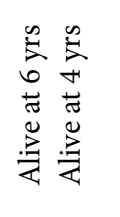 & 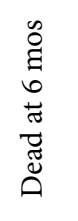 & 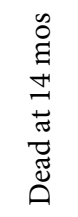 & 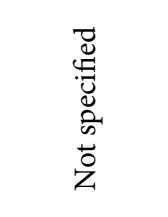 & 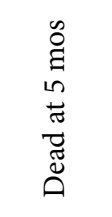 & 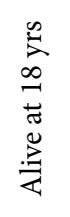 & 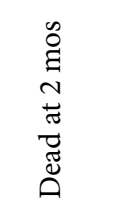 & 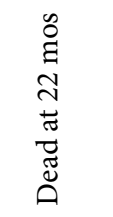 & $\begin{array}{l}0 \\
0 \\
0 \\
0 \\
0 \\
0 \\
\tilde{J} \\
\tilde{\Xi} \\
0\end{array}$ & 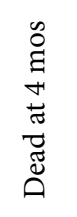 & 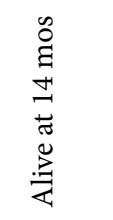 & 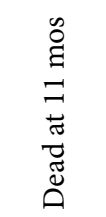 & 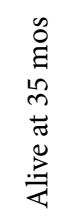 & 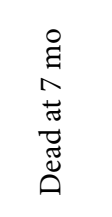 & 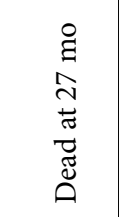 \\
\hline 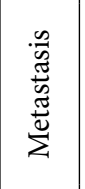 & z & 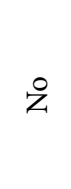 & $\stackrel{\circ}{z} \stackrel{\circ}{ }$ & 品 & $\stackrel{\infty}{\Xi}$ & $\stackrel{\circ}{z}$ & 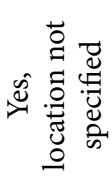 & $\stackrel{2}{z}$ & 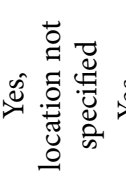 & 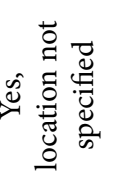 & 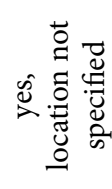 & 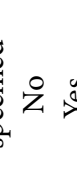 & 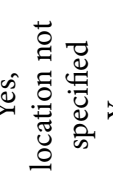 & 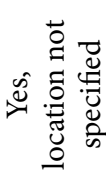 & $\sum_{2}^{0}$ & 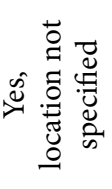 & 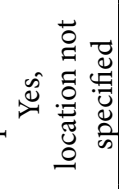 \\
\hline 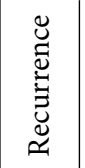 & z & ஜ & $\stackrel{\overbrace{}}{\check{z}}$ & $\cong$ & $\cong$ & 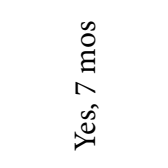 & 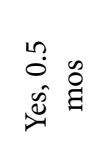 & z̊ & $\dot{z}$ & $\begin{array}{l}0 \\
\stackrel{\Xi}{1} \\
m \\
\hat{ٌ}\end{array}$ & 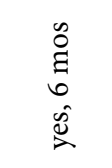 & 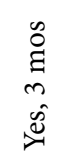 & 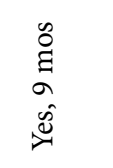 & 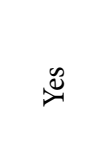 & $\stackrel{\circ}{z}$ & 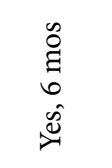 & 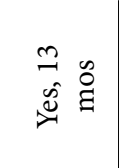 \\
\hline 苋 & z̊ & $\stackrel{\circ}{z}$ & $\stackrel{2}{z}$ & ż & $\stackrel{0}{z}$ & $\stackrel{0}{z}$ & $\triangleq$ & 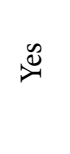 & ż & 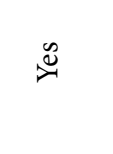 & $\cong$ & $\cong$ & ż & 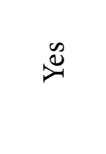 & $\stackrel{\circ}{z}$ & z & $\stackrel{2}{z}$ \\
\hline 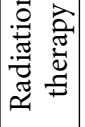 & ż & ஜ & $\stackrel{0}{z}$ & 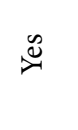 & 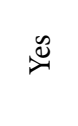 & z & $\stackrel{\Xi}{\approx}$ & $\approx$ & ż & $\approx$ & $\stackrel{\infty}{2}$ & z & ż & $\stackrel{\circ}{z}$ & $\ddot{z}$ & $\stackrel{2}{z}$ & $\approx$ \\
\hline 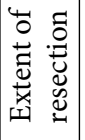 & 胥 & 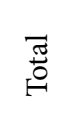 & 吾 & 胥 & 点 & 吾 & 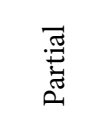 & 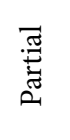 & $\underset{ت}{\vec{\Xi}}$ & 烝 & $\begin{array}{l}\stackrel{\vec{Z}}{\vec{Z}} \\
\tilde{a}\end{array}$ & 烝 & 푱 & 苟 & ㅍّㅁ & 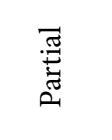 & 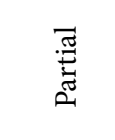 \\
\hline 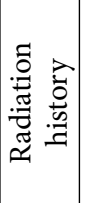 & 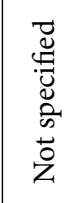 & 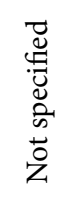 & 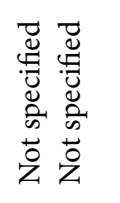 & 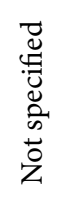 & 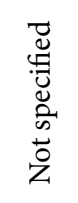 & 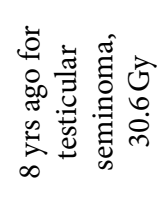 & 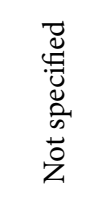 & 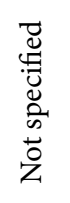 & 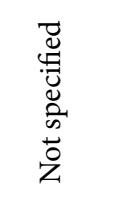 & 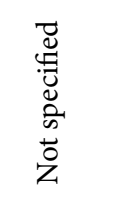 & 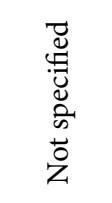 & 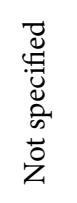 & 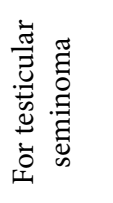 & 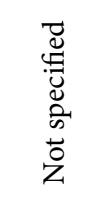 & 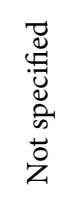 & 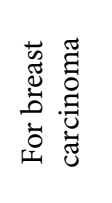 & 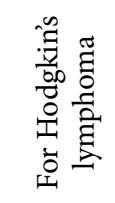 \\
\hline$\frac{0}{.00}$ & & & & 豆 & & & 豆 & 豆 & $\overrightarrow{\mathrm{I}}$ & $\overrightarrow{\mathrm{Z}}$ & $\overrightarrow{\mathrm{I}}$ & & & $\overline{\mathrm{Z}}$ & $\overrightarrow{\mathrm{Z}}$ & & \\
\hline 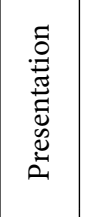 & 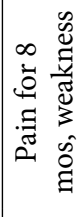 & 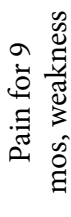 & 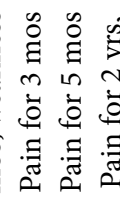 & 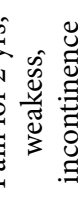 & 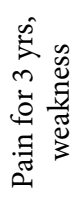 & 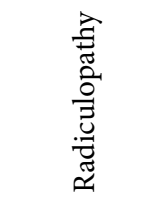 & 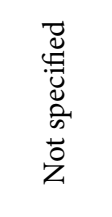 & 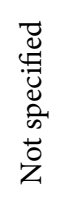 & 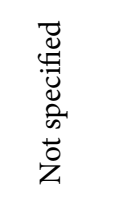 & 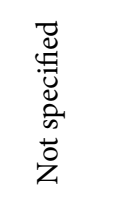 & 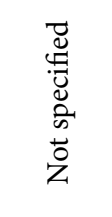 & 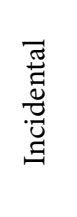 & 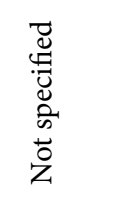 & 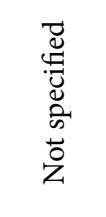 & 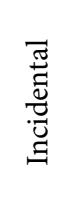 & 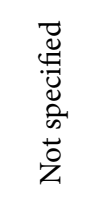 & 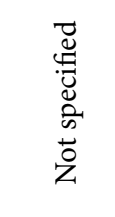 \\
\hline 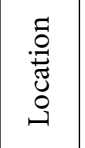 & 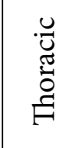 & $\begin{array}{l}\dot{\Xi} \\
\stackrel{\Xi}{\Xi}\end{array}$ & 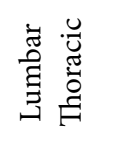 & Uू. & 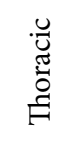 & ت্ّ̆ & $\begin{array}{l}\hat{\tilde{E}} \\
\stackrel{\vec{J}}{\breve{Z}} \\
\underline{\Xi}\end{array}$ & 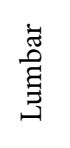 & 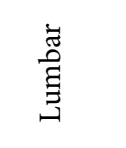 & 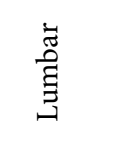 & 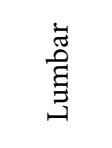 & 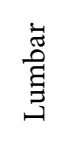 & 苛 & 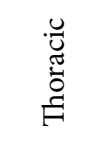 & $\begin{array}{l}\stackrel{\breve{G}}{\tilde{J}} \\
\tilde{E}\end{array}$ & 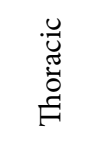 & 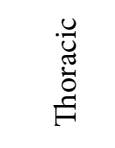 \\
\hline 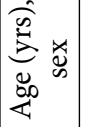 & $\begin{array}{l}\vec{w} \\
\hat{n}\end{array}$ & $\begin{array}{l}\stackrel{4}{0} \\
\infty \hat{0}\end{array}$ & 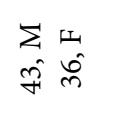 & $\begin{array}{l}\vec{w} \\
\hat{\imath}\end{array}$ & $\begin{array}{l}\sum \\
0 \\
\hat{m}\end{array}$ & $\begin{array}{l}\sum \\
0 \\
0\end{array}$ & $\stackrel{\vec{w}}{=}$ & 讪 & $\hat{m}$ & $\begin{array}{l}\sum \\
\hat{m}\end{array}$ & $\stackrel{\vec{m}}{\vec{m}}$ & 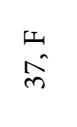 & $\begin{array}{l}\Sigma \\
0 \\
\hat{q}\end{array}$ & $\begin{array}{l}\Sigma \\
\Omega\end{array}$ & $\stackrel{a}{a}$ & $\stackrel{4}{\hat{n}}$ & $\begin{array}{l}\Sigma \\
0 \\
0\end{array}$ \\
\hline 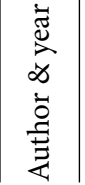 & & & 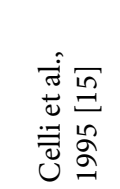 & & & 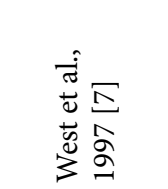 & & & & & & 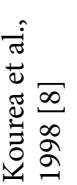 & & & & & \\
\hline
\end{tabular}




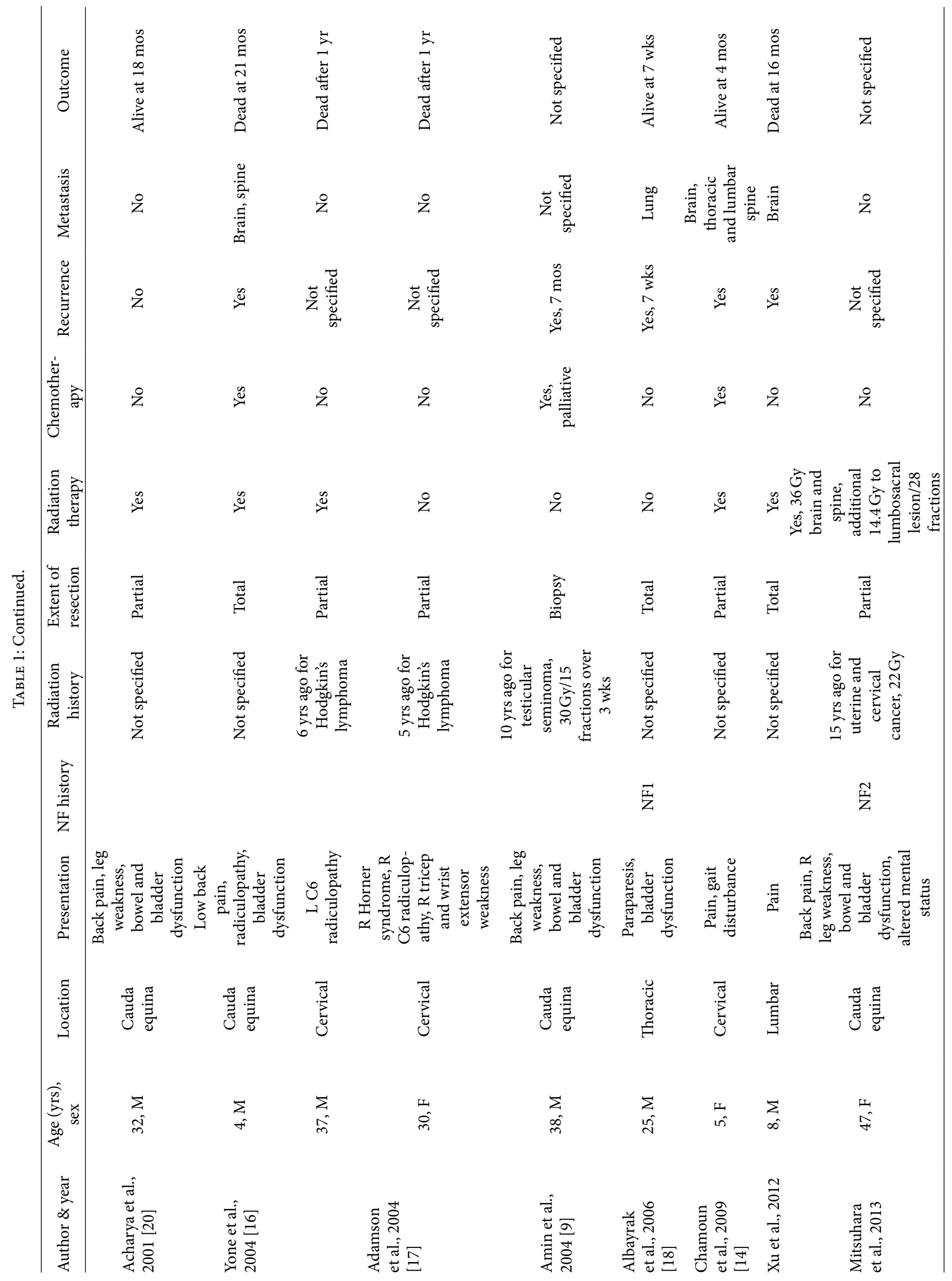




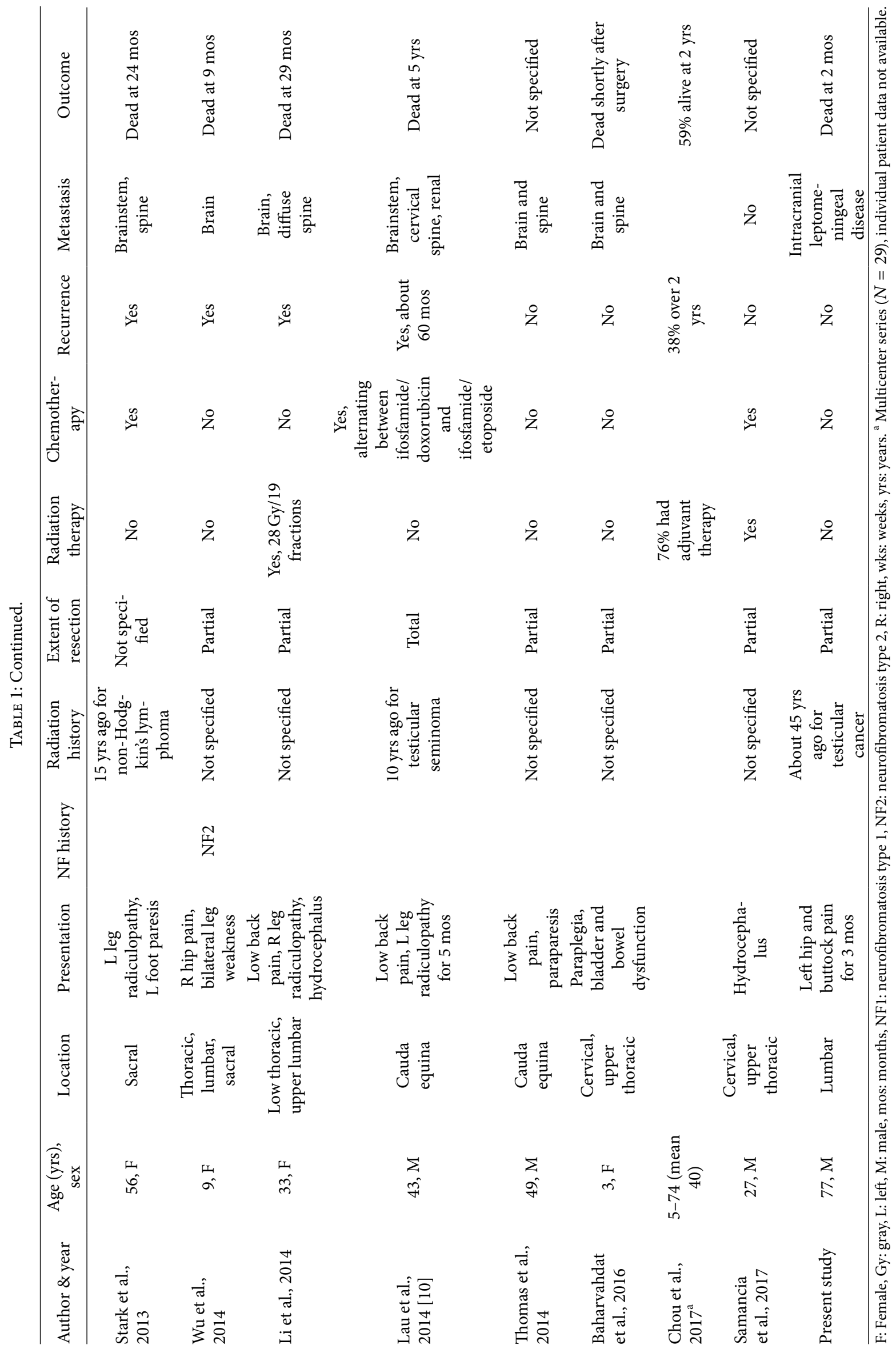




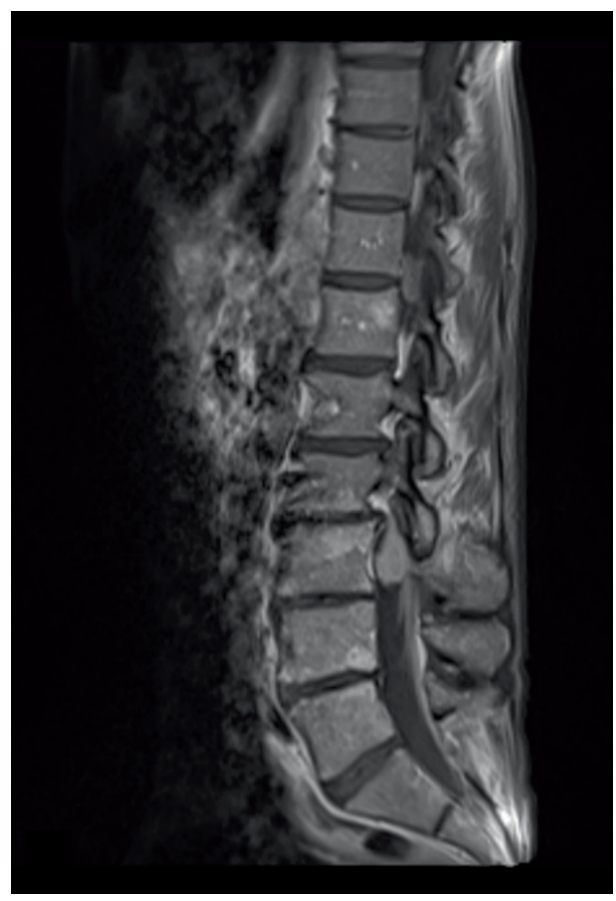

(a)

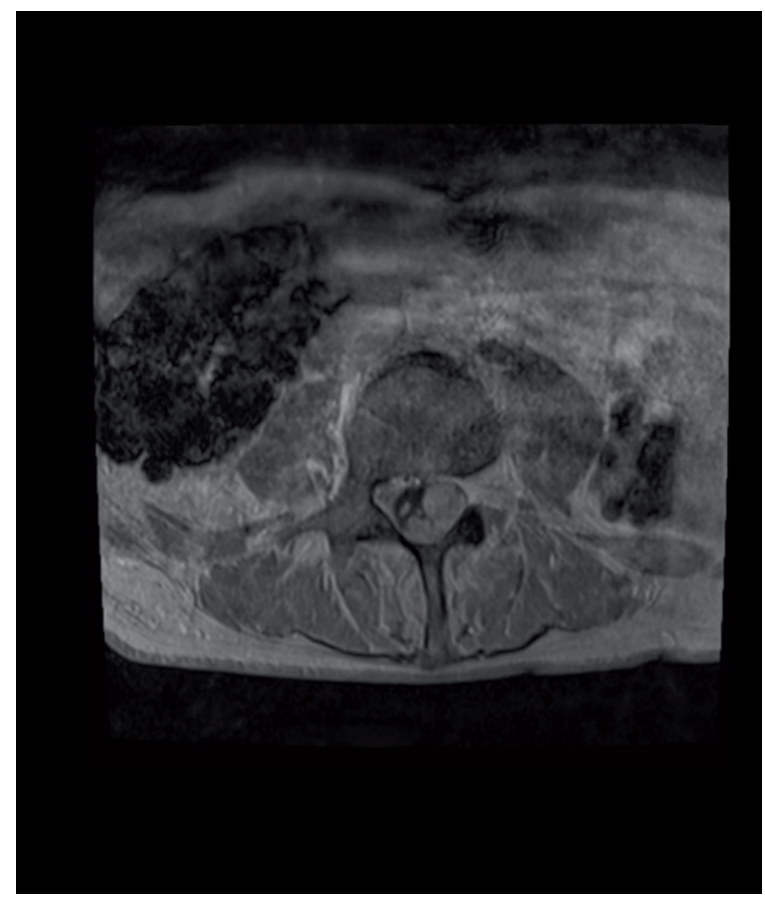

(b)

FIGURE 1: (a) Magnetic resonance imaging (MRI) T1-weighted sagittal image of the lumbar spine with gadolinium demonstrates a well demarcated intradural extramedullary mass. (b) Corresponding axial image.

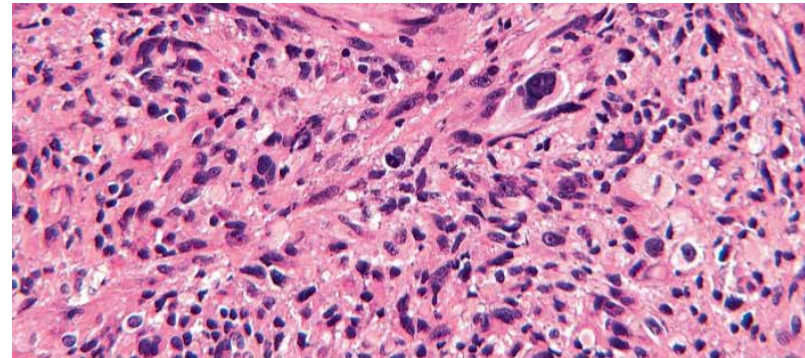

(a)

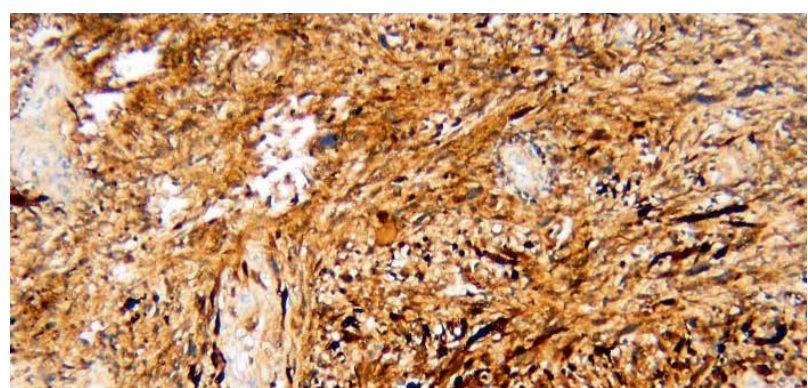

(c)

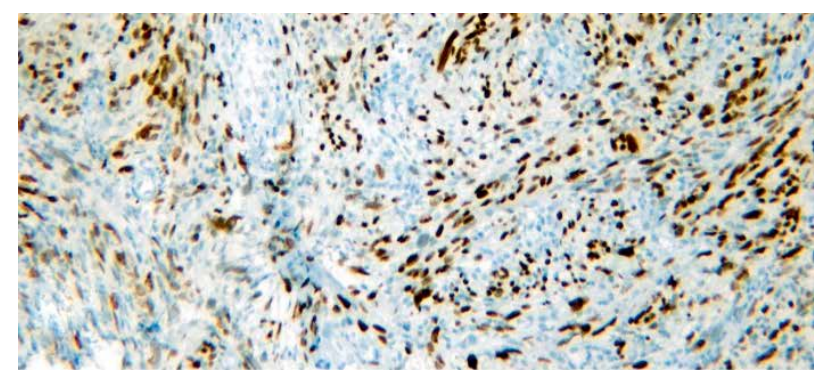

(b)

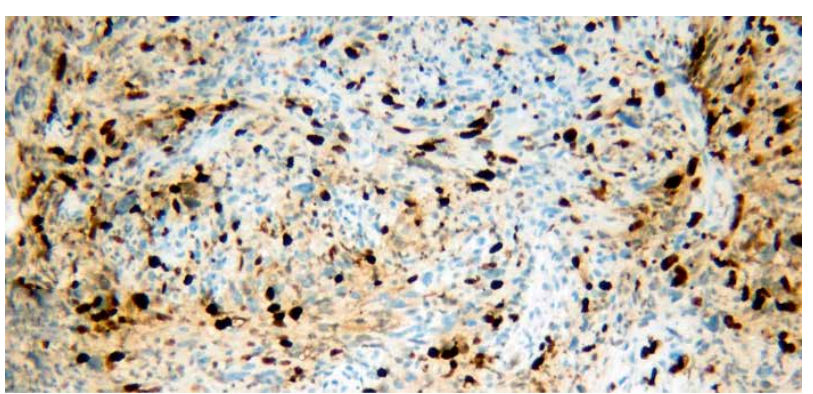

(d)

FIGURE 2: (a) Hematoxylin and eosin (H\&E) staining showed high cellular density with marked pleomorphism and spindle cells arranged in fascicles (200x). (b) Immunohistochemistry was positive for SOX 10 (100x) and (c) S-100 (100x). (d) Ki-67 labeling revealed a high proliferative index $(100 x)$.

history of cancer. A metastatic work up performed to look for any additional lesions was negative.

2.2. Operative Course. A partial L2 and complete L3 laminectomy was performed and a midline durotomy was made. A greyish mass was found in the intradural space arising from the left L3 nerve root with obvious enlargement and involvement of the nerve root. A frozen specimen was sent early for pathological evaluation that was diagnosed as MPNST. The nerve root of interest was clearly identified both proximally and distally, which on stimulation resulted in robust electrophysiological response suggestive of origin 


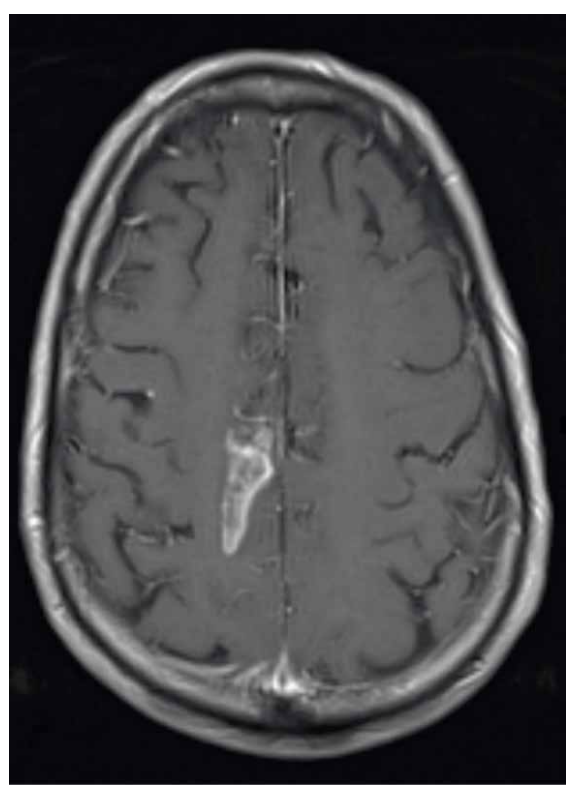

(a)

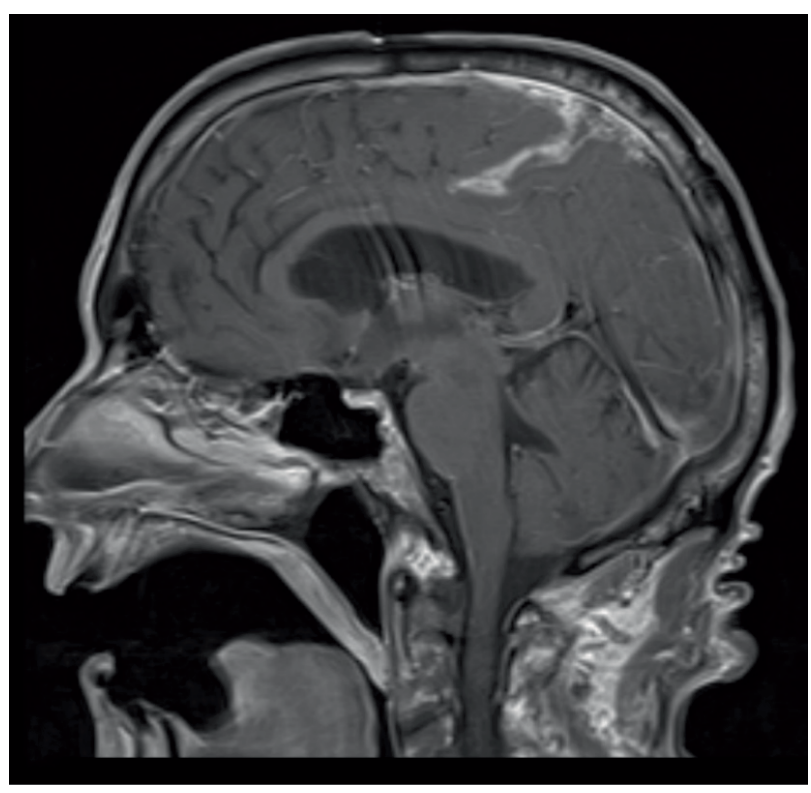

(b)

FIgURE 3: (a) Magnetic resonance imaging (MRI) T1-weighted axial image of the brain with gadolinium demonstrates leptomeningeal metastasis. (b) Corresponding sagittal image.

from a motor nerve root. No obvious plane was found between the tumor and the nerve root. Given the risk of motor deficit, the nerve root was preserved with partial resection of the tumor. Final pathology confirmed the diagnosis of MPNST (Figure 2).

2.3. Post-Operative Course. Given the initial pathologic diagnosis on frozen section, subsequent management including reoperation with extensive resection was discussed. Given his preoperative status, the patient, and family elected to observe in the short term with consideration of further treatment options following final pathology. The patient did well initially and was discharged to a skilled nursing facility. Two weeks post-operatively, however, the patient was readmitted with encephalopathy. An MRI brain with and without gadolinium demonstrated leptomeningeal metastasis (Figure 3) with cerebral spinal fluid (CSF) cytology positive for malignant cells. Given the extent of disseminated disease and his progressive worsening mental status, the family elected to pursue palliative care, and the patient died two months after his initial surgery.

\section{Discussion}

MPNSTs are rare entities with an incidence of $0.001 \%$, with $20-50 \%$ of cases arising in patients with NF- 1 . The most common locations include the trunk, extremities, and head, and neck [2]. Primary spinal MPNSTs are extremely rare. Primary MPNSTs of the spine that are exclusively intradural extramedullary without extension into the extradural compartment are exceptionally rare. Outside the setting of NF-1, prior radiation treatment is a risk factor for development of MPNSTs. There have been few reports of MPNST following prior radiation for testicular seminomas. Most occurred after a short latent period and in an extradural location, with a purely intradural occurrence being exceptionally rare [7-10].

The present case developed almost four decades after being treated with EBRT for testicular seminoma, which is a significantly longer latency period as compared to other cases previously reported. Also, the imaging features were fairly characteristic of a benign intradural extramedullary neoplasm unlike a MPNST, which is typically an irregularly-bordered heterogeneously enhancing mass often with destruction of surrounding osseous structures. Similarly, the presence of leptomeningeal spread is also rare in primary intradural MPNSTs and as illustrated in this case, portends an ominous prognosis [11]. Unique to this case, however, is the rapid development of disseminated disease two weeks after surgery. Patients in other reported cases of intradural MPNSTs with leptomeningeal spread were diagnosed with dissemination ranging from present on presentation to 24 months following initial surgery [10-19]. The authors advocate that regardless of imaging characteristics or the duration since radiation, surgeons should retain a high index of suspicion for a MPSNT. Lumbar puncture may be considered to obtain CSF to identify potential malignant cells.

\section{Conclusion}

A rare case of an intradural MPNST diagnosed more than 40 years after radiation for a testicular seminoma is reported. Intradural MPNSTs of the spine outside the setting of neurofibromatosis are extremely rare and can masquerade common benign nerve sheath tumors on imaging. Short duration of symptoms and prior regional radiation treatment encompassing the spine in the treatment field regardless of remoteness should alert the physician to the possible existence of this rare 
and aggressive tumor. Being cognizant of this rare pathology can help initiate appropriate work up and evaluation, allow preoperative counselling, and alter overall surgical strategy.

\section{Abbreviations}

$\begin{array}{ll}\text { CSF: } & \text { Cerebral spinal fluid } \\ \text { CT: } & \text { Computer tomography } \\ \text { EBRT: } & \text { External bean radiation therapy } \\ \text { H\&E: } & \text { Hematoxylin and eosin } \\ \text { MPNST: } & \text { Malignant peripheral nerve sheath tumor } \\ \text { MRI: } & \text { Magnetic resonance imaging } \\ \text { NF1: } & \text { Neurofibromatosis type } 1 .\end{array}$

\section{Conflicts of Interest}

The authors declare that they have no conflicts of interest.

\section{References}

[1] A. N. D’Agostino, E. H. Soule, and R. H. Miller, "Sarcomas of the peripheral nerves and somatic soft tissues associated with multiple neurofibromatosis (Von Recklinghausen's disease)," Cancer, vol. 16, no. 8, pp. 1015-1027, 1963.

[2] B. S. Ducatman, B. W. Scheithauer, D. G. Piepgras, H. M. Reiman, and D. M. Ilstrup, "Malignant peripheral nerve sheath tumors. A clinicopathologic study of 120 cases," Cancer, vol. 57, no. 10, pp. 2006-2021, 1986.

[3] S. I. Hajdu, "Peripheral nerve sheath tumors histogenesis, classification, and prognosis," Cancer, vol. 72, no. 12, pp. 3549-3552, 1993.

[4] B. Zhu, X. Liu, Z. Liu et al., "Malignant peripheral nerve sheath tumours of the spine: clinical manifestations, classification, treatment, and prognostic factors," European Spine Journal, vol. 21, no. 5, pp. 897-904, 2012.

[5] D. G. Evans, M. E. Baser, J. McGaughran, S. Sharif, E. Howard, and A. Moran, "Malignant peripheral nerve sheath tumours in neurofibromatosis 1," Journal of Medical Genetics, vol. 39, no. 5, pp. 311-314, 2002

[6] G. Gupta and A. Maniker, "Malignant peripheral nerve sheath tumors," Neurosurgical Focus, vol. 22, no. 6, pp. 1-8, 2007.

[7] D. A. West, R. O. Parra, A. Manepalli, R. J. Bernardi, and J. M. Cummings, "Development of a malignant peripheral nerve sheath tumor following treatment for testicular seminoma," Urology, vol. 50, no. 2, pp. 292-294, 1997.

[8] H. P. Kourea, M. H. Bilsky, D. H. Leung, J. J. Lewis, and J. M. Woodruff, "Subdiaphragmatic and intrathoracic paraspinal malignant peripheral nerve sheath tumors: a clinicopathologic study of 25 patients and 26 tumors," Cancer, vol. 82, no. 11, pp. 2191-2203, 1998.

[9] A. Amin, A. Saifuddin, A. Flanagan, D. Patterson, and J. Lehovsky, "Radiotherapy-induced malignant peripheral nerve sheath tumor of the cauda equina," Spine, vol. 29, no. 21, pp. E506-E509, 2004.

[10] D. Lau, D. H. Moon, P. Park, S. Hervey-Jumper, P. E. McKeever, and D. A. Orringer, "Radiation-induced intradural malignant peripheral nerve sheath tumor of the cauda equina with diffuse leptomeningeal metastasis," Journal of Neurosurgery: Spine, vol. 21, no. 5, pp. 719-726, 2014.

[11] A. M. Stark and H. M. Mehdorn, "Leptomeningeal metastasis of an intradural malignant peripheral nerve sheath tumor," Journal of Clinical Neuroscience, vol. 20, no. 8, pp. 1181-1183, 2013.

[12] J. M. Valdueza, C. Hagel, M. Westphal, M. Hänsel, and H. D. Herrmann, "Primary spinal malignant schwannoma: clinical, histological and cytogenetic findings," Neurosurgical Review, vol. 14, no. 4, pp. 283-291, 1991.

[13] K. Yone, K. Ijiri, K. Hayashi et al., "Primary malignant peripheral nerve sheath tumor of the cauda equina in a child case report," Spinal Cord, vol. 42, no. 3, pp. 199-203, 2004.

[14] R. B. Chamoun, W. E. Whitehead, R. C. Dauser et al., "Primary disseminated intradural malignant peripheral nerve sheath tumor of the spine in a child: case report and review of the literature," Pediatric Neurosurgery, vol. 45, no. 3, pp. 230-236, 2009.

[15] Q. Xu, B. Xing, X. Huang, R. Wang, Y. Li, and Z. Yang, "Primary malignant peripheral nerve sheath tumor of the cauda equina with metastasis to the brain in a child: case report and literature review," The Spine Journal, vol. 12, no. 4, pp. e7-e13, 2012.

[16] L. Wu, X. Deng, C. Yang, and Y. Xu, "Spinal intradural malignant peripheral nerve sheath tumor in a child with neurofibromatosis type 2: the first reported case and literature review," Turkish Neurosurgery, vol. 24, no. 1, pp. 135-139, 2014.

[17] Y. Li, F. Fan, J. Xu, J. An, and W. Zhang, "Primary malignant peripheral nerve sheath tumor of the spine with acute hydrocephalus: a rare clinical entity," Journal of Neurosurgery: Spine, vol. 21, no. 3, pp. 367-371, 2014.

[18] J. G. Thomas, C. Lincoln, J. C. Goodman, and S. P. Gopinath, "Malignant peripheral nerve sheath tumor of the cauda equina with craniospinal metastasis," Journal of Clinical Neuroscience, vol. 21, no. 12, pp. 2239-2242, 2014.

[19] H. Baharvahdat, B. Ganjeifar, N. Mohamadian Roshan, and A. Baradaran, "Spinal intradural primary malignant peripheral nerve sheath tumor with leptomeningeal seeding: case report and literature review," Turkish Neurosurgery, vol. 28, no. 2, pp. 317-322, 2018.

[20] R. Acharya, S. Bhalla, and A. Sehgal, "Malignant peripheral nerve sheath tumor of the cauda equina," Neurological Sciences, vol. 22, no. 3, pp. 267-270, 2001. 